\title{
Minimax Interpolation Problem for Random Processes with Stationary Increments
}

\author{
Maksym Luz $^{1}$, Mikhail Moklyachuk ${ }^{1, *}$ \\ ${ }^{1}$ Department of Probability Theory, Statistics and Actuarial Mathematics, Taras Shevchenko National University of Kyiv, Ukraine
}

Received: 20 September 2014; Accepted: 10 February 2015

Editor: Tatiana Tchemisova

\begin{abstract}
The problem of mean-square optimal estimation of the linear functional $A_{T} \xi=\int_{0}^{T} a(t) \xi(t) d t$ that depends on the unknown values of a continuous time random process $\xi(t), t \in \mathbb{R}$, with stationary $n$th increments from observations of the process $\xi(t)$ at time points $t \in \mathbb{R} \backslash[0 ; T]$ is investigated under the condition of spectral certainty as well as under the condition of spectral uncertainty. Formulas for calculation the value of the mean-square error and spectral characteristic of the optimal linear estimate of the functional are derived under the condition of spectral certainty where spectral density of the process is exactly known. In the case of spectral uncertainty where spectral density of the process is not exactly known, but a class of admissible spectral densities is given, relations that determine the least favourable spectral density and the minimax spectral characteristic are specified.
\end{abstract}

Keywords Random process with stationary increments, minimax-robust estimate, mean-square error, least favourable spectral density, minimax spectral characteristic

AMS 2010 subject classifications Primary: 60G10, 60G25, 60G35, Secondary: 62M20, 93E10, 93E11

DOI: $10.19139 /$ soic.v3i1.105

\section{Introduction}

The classical methods of finding solutions to extrapolation, interpolation and filtering problems for stationary random processes and sequences were developed by Kolmogorov [12], Wiener [35], Yaglom [36, 37]. In the paper [38] Yaglom generalized the theory of continuous time stationary processes to the case of random processes with stationary $n$th increments. He found spectral representation of the increment process, canonical factorization of spectral density and solved the extrapolation problem for random processes with stationary increments. For more details one can see papers by Yaglom and Pinsker [30], Pinsker [31]. A few years later Yaglom [39] applied the theory of generalized random process to investigation random processes with stationary increments.

Most of results concerning estimation of the unknown (missed) values of random processes are based on the assumption that spectral densities of random processes are exactly known. In practice, however, complete information on the spectral densities is impossible in most cases. In such situations one finds parametric or nonparametric estimates of the unknown spectral densities. Then the classical estimation method is applied under the assumption that the estimated densities are true. This procedure can result in significant increasing of the value of error of estimate as Vastola and Poor [34] have demonstrated with the help of some examples. This is a reason to search estimates which are optimal for all densities from a certain class of admissible spectral densities. These

\footnotetext{
*Correspondence to: Mikhail Moklyachuk (Email: Moklyachuk@gmail.com). Department of Probability Theory, Statistics and Actuarial Mathematics, Taras Shevchenko National University of Kyiv, Volodymyrska 64 Str., Kyiv 01601, Ukraine.
}

ISSN 2310-5070 (online) ISSN 2311-004X (print)

Copyright (C) 2015 International Academic Press 
estimates are called minimax since they minimize the maximal value of the error of estimate. A survey of results in minimax (robust) methods of data processing can be found in paper by Kassam and Poor [11]. The paper by Grenander [7] should be marked as the first one where the minimax extrapolation problem for continuous time stationary processes was formulated and solved. Later Franke [8], Franke and Poor [9] investigated the minimaxrobust extrapolation and interpolation problems for stationary sequences by using methods of convex optimization. In papers by Moklyachuk [19] - [26] the minimax approach was applied to extrapolation, interpolation and filtering problems for functionals which depend on the unknown values of stationary processes. Methods of solution the minimax-robust estimation problems for vector-valued stationary processes were developed by Moklyachuk and Masyutka [23] - [28]. Analogous minimax estimation problems (extrapolation, interpolation and filtering) for linear functionals which depend on unknown values of periodically correlated stochastic processes were investigated by Dubovets'ka and Moklyachuk [2] - [6]. Minimax-robust extrapolation, interpolation and filtering problems for random processes and sequences with stationary increments are investigated by Luz and Moklyachuk [13] [18]. In particular, solutions to the minimax-robust interpolation problem for stochastic sequences with stationary increments are proposed in papers [13],[14].

In this paper we investigate the problem of mean-square optimal estimation of the linear functional $A_{T} \xi=$ $\int_{0}^{T} a(t) \xi(t) d t$ that depends on the unknown values of a continuous time random process $\xi(t), t \in \mathbb{R}$, with stationary $n$th increments from observations of this process at time points $t \in \mathbb{R} \backslash[0 ; T]$. Formulas for calculating the value of the mean-square error and the spectral characteristic of the optimal estimate of the functional $A_{T} \xi$ are derived under the condition of spectral certainty where spectral density of the process is exactly known. In the case where spectral density is not known, but a set of admissible spectral densities is available, relations which determine least favourable spectral densities and the minimax-robust spectral characteristics for different classes of spectral densities are specified.

\section{Stationary increment random process. Spectral representation}

In this section we present a brief review of spectral properties of random processes with stationary increment. For more detailed information see articles and books [30], [31], [36] - [38].

\section{Definition 1}

For a given random process $\{\xi(t), t \in \mathbb{R}\}$ the process

$$
\xi^{(n)}(t, \tau)=\left(1-B_{\tau}\right)^{n} \xi(t)=\sum_{l=0}^{n}(-1)^{l} C_{n}^{l} \xi(t-l \tau),
$$

where $B_{\tau}$ is a backward shift operator with a step $\tau \in \mathbb{R}$ such that $B_{\tau} \xi(t)=\xi(t-\tau)$, is called random $n$th increment process with step $\tau \in \mathbb{R}$.

The random $n$th increment process $\xi^{(n)}(t, \tau)$ satisfies the relations

$$
\begin{gathered}
\xi^{(n)}(t,-\tau)=(-1)^{n} \xi^{(n)}(t+n \tau, \tau), \\
\xi^{(n)}(t, k \tau)=\sum_{l=0}^{(k-1) n} A_{l} \xi^{(n)}(t-l \tau, \tau), \quad \forall k \in \mathbb{N},
\end{gathered}
$$

with coefficients $\left\{A_{l}, l=0,1,2, \ldots,(k-1) n\right\}$ determined from the representation

$$
\left(1+x+\ldots+x^{k-1}\right)^{n}=\sum_{l=0}^{(k-1) n} A_{l} x^{l} .
$$

Definition 2

The random $n$th increment process $\xi^{(n)}(t, \tau)$ generated by a random process $\{\xi(t), t \in R\}$ is wide sense stationary 
if the mathematical expectations

$$
\begin{gathered}
\mathrm{E} \xi^{(n)}\left(t_{0}, \tau\right)=c^{(n)}(\tau), \\
\mathrm{E} \xi^{(n)}\left(t_{0}+t, \tau_{1}\right) \xi^{(n)}\left(t_{0}, \tau_{2}\right)=D^{(n)}\left(t, \tau_{1}, \tau_{2}\right)
\end{gathered}
$$

exist for all $t_{0}, \tau, t, \tau_{1}, \tau_{2}$ and do not depend on $t_{0}$. The function $c^{(n)}(\tau)$ is called the mean value of the $n$th increment process and the function $D^{(n)}\left(t, \tau_{1}, \tau_{2}\right)$ is called the structural function of the stationary $n$th increment process (or the structural function of $n$th order of the random process $\{\xi(t), t \in \mathbb{R}\}$ ).

The random process $\{\xi(t), t \in \mathbb{R}\}$ which determines the stationary $n$th increment process $\xi^{(n)}(t, \tau)$ by formula (1) is called random process with stationary $n$th increments.

\section{Theorem 1}

The mean value $c^{(n)}(\tau)$ and the structural function $D^{(n)}\left(t, \tau_{1}, \tau_{2}\right)$ of a random stationary $n$th increment process $\xi^{(n)}(t, \tau)$ can be represented in the following forms

$$
\begin{gathered}
c^{(n)}(\tau)=c \tau^{n}, \\
D^{(n)}\left(t, \tau_{1}, \tau_{2}\right)=\int_{-\infty}^{\infty} e^{i \lambda t}\left(1-e^{-i \tau_{1} \lambda}\right)^{n}\left(1-e^{i \tau_{2} \lambda}\right)^{n} \frac{\left(1+\lambda^{2}\right)^{n}}{\lambda^{2 n}} d F(\lambda),
\end{gathered}
$$

where $c$ is a constant, $F(\lambda)$ is a left-continuous nondecreasing bounded function with $F(-\infty)=0$. The constant $c$ and the spectral function $F(\lambda)$ are determined uniquely by the increment process $\xi^{(n)}(t, \tau)$.

On the other hand, a function $c^{(n)}(\tau)$ which has form (4) with a constant $c$ and a function $D^{(n)}\left(t, \tau_{1}, \tau_{2}\right)$ which has form (5) with a function $F(\lambda)$ which satisfies the indicated conditions are the mean value and the structural function of some stationary $n$th increment process $\xi^{(n)}(t, \tau)$.

Using representation (5) of the structural function of the stationary $n$th increment process $\xi^{(n)}(t, \tau)$ and the Karhunen theorem (see Karhunen [10]), we get the following spectral representation of the stationary $n$th increment process $\xi^{(n)}(t, \tau)$ :

$$
\xi^{(n)}(t, \tau)=\int_{-\infty}^{\infty} e^{i t \lambda}\left(1-e^{-i \lambda \tau}\right)^{n} \frac{(1+i \lambda)^{n}}{(i \lambda)^{n}} d Z(\lambda),
$$

where $Z(\lambda)$ is a random process with independent increments on $\mathbb{R}$ connected with the spectral function $F(\lambda)$ by the relation

$$
\mathrm{E}\left|Z\left(t_{2}\right)-Z\left(t_{1}\right)\right|^{2}=F\left(t_{2}\right)-F\left(t_{1}\right)<\infty \quad \text { for all } t_{2}>t_{1} .
$$

In the following we will consider increments $\xi^{(n)}(t, \tau)$ with step $\tau>0$ and mean value 0 .

\section{Interpolation problem}

Consider a random process $\{\xi(t), t \in \mathbb{R}\}$ which determines a stationary $n$th increment $\xi^{(n)}(t, \tau)$ with an absolutely continuous spectral function $F(\lambda)$ which has spectral density $f(\lambda)$ satisfying the minimality conditions:

$$
\int_{-\infty}^{+\infty} \frac{|\gamma(\lambda)|^{2}}{f(\lambda)} d \lambda<\infty, \quad \int_{-\infty}^{+\infty} \frac{|\gamma(\lambda)|^{2} \lambda^{2 n}}{\left|1-e^{i \lambda \tau}\right|^{2 n}\left(1+\lambda^{2}\right)^{n} f(\lambda)} d \lambda<\infty
$$

for all non-zero function of the exponential type $\gamma(\lambda)=\int_{0}^{T+\tau n} \alpha(t) e^{i \lambda t} d t$.

Suppose that we observe values of the process $\xi(t)$ at time points $t \in \mathbb{R} \backslash[0, T]$. The interpolation problem consists in finding the mean-square optimal linear estimate of the functional $A_{T} \xi=\int_{0}^{T} a(t) \xi(t) d t$ which depends on the unknown values of the considered process $\xi(t)$. Denote this estimate by $\widehat{A}_{T} \xi$.

In this article we use an approach to solution the interpolation problem which consists in representation of the functional $A_{T} \xi=\int_{0}^{T} a(t) \xi(t) d t$ as a difference of two functionals, one of which depends on increments $\xi^{(n)}(t, \tau)$, $t>0$, for a fixed $\tau>0$, and the other one depends on the observed values of the process $\xi(t)$. Such a representation, which is obtained in [16], is described in the following lemma. 
Lemma 1

The linear functional $A_{T} \xi=\int_{0}^{T} a(t) \xi(t) d t$ admits the representation

$$
\begin{gathered}
A_{T} \xi=B_{T} \xi-V_{T} \xi \\
B_{T} \xi=\int_{0}^{T} b_{\tau, T}(t) \xi^{(n)}(t, \tau) d t, \quad V_{T} \xi=\int_{-\tau n}^{0} v_{\tau, T}(t) \xi(t) d t \\
v_{\tau, T}(t)=\sum_{l=\left[-\frac{t}{\tau}\right]^{\prime}}^{\min \left\{\left[\frac{T-t}{\tau}\right], n\right\}}(-1)^{l} C_{n}^{l} b_{\tau, T}(l \tau+t), \quad t \in[-\tau n ; 0), \\
b_{\tau, T}(t)=\sum_{k=0}^{\left[\frac{T-t}{\tau}\right]} a(t+\tau k) d(k)=\mathbf{D}_{T}^{\tau} a(t), \quad t \in[0 ; T],
\end{gathered}
$$

where $[x]^{\prime}$ denotes the least integer number among numbers greater than or equal to $x,\{d(k): k \geq 0\}$ are coefficients determined by the relation $\sum_{k=0}^{\infty} d(k) x^{k}=\left(\sum_{j=0}^{\infty} x^{j}\right)^{n}, \mathbf{D}_{T}^{\tau}$ is a linear transformation which acts on arbitrary function $x(t), t \in[0, T]$, in the following way:

$$
\mathbf{D}_{T}^{\tau} x(t)=\sum_{k=0}^{\left[\frac{T-t}{\tau}\right]} x(t+\tau k) d(k) .
$$

Having observations of the random process $\xi(t)$ at time points $t \in \mathbb{R} \backslash[0 ; T]$ we get values of the increment process $\xi^{(n)}(t, \tau)$ at points $t \in \mathbb{R} \backslash[0 ; T+\tau n]$ by formula (1) and construct a mean-square optimal estimate $\widehat{B}_{T} \xi$ of the functional $B_{T} \xi$ based on these observations. We conclude from Lemma 1 that

$$
\widehat{A}_{T} \xi=\widehat{B}_{T} \xi-V_{T} \xi
$$

and the mean square errors $\Delta\left(f, \widehat{A}_{T} \xi\right)=\mathrm{E}\left|A_{T} \xi-\widehat{A}_{T} \xi\right|^{2}$ and $\Delta\left(f, \widehat{B}_{T} \xi\right)=\mathrm{E}\left|B_{T} \xi-\widehat{B}_{T} \xi\right|^{2}$ of the estimates $\widehat{A}_{T} \xi$ and $\widehat{B}_{T} \xi$ satisfy the following equalities

$$
\Delta\left(f, \widehat{A}_{T} \xi\right)=\mathrm{E}\left|A_{T} \xi-\widehat{A}_{T} \xi\right|^{2}=\mathrm{E}\left|A_{T} \xi+V_{T} \xi-\widehat{B}_{T} \xi\right|^{2}=\mathrm{E}\left|B_{T} \xi-\widehat{B}_{T} \xi\right|^{2}=\Delta\left(f, \widehat{B}_{T} \xi\right)
$$

So we have to find the optimal estimate $\widehat{B}_{T} \xi$ of the linear functional $B_{T} \xi$ in order to solve the interpolation problem for the linear functional $A_{T} \xi$.

Every linear estimate $\widehat{B}_{T} \xi$ of the linear functional $B_{T} \xi$ admits the spectral representation

$$
\widehat{B}_{T} \xi=\int_{-\infty}^{+\infty} h_{\tau}(\lambda) d Z(\lambda),
$$

where $h_{\tau}(\lambda)$ is the spectral characteristic of the estimate. This spectral characteristic can be found by using the Hilbert space orthogonal projection method proposed by Kolmogorov [12].

Let us define some spaces associated with the increment process $\xi^{(n)}(t, \tau)$. Let $H^{0-}\left(\xi_{\tau}^{(n)}\right)$ denote a closed linear subspace in the space $H=L_{2}(\Omega, \mathcal{F}, P)$ of second order random variables generated by $n$th increments $\left\{\xi^{(n)}(t, \tau): t<0\right\}$ and let $H^{T+}\left(\xi_{-\tau}^{(n)}\right)$ denote a closed linear subspace in the space $H=L_{2}(\Omega, \mathcal{F}, P)$ generated by $n$th increments $\left\{\xi^{(n)}(t,-\tau): t>T\right\}$. It follows from the equality

$$
e^{i \lambda t}\left(1-e^{i \lambda \tau}\right)^{n}=(-1)^{n} e^{i \lambda(t+\tau n)}\left(1-e^{-i \lambda \tau}\right)^{n}
$$


that $\xi^{(n)}(t,-\tau)=(-1)^{n} \xi^{(n)}(t+\tau n, \tau)$. Therefore, $H^{T+}\left(\xi_{-\tau}^{(n)}\right)=H^{(T+\tau n)+}\left(\xi_{\tau}^{(n)}\right)$. Let us define subspaces $L_{2}^{0-}(f)$ and $L_{2}^{T+}(f)$ of the Hilbert space $L_{2}(f)$ which are generated by the sets of functions

$$
\left\{e^{i \lambda t}\left(1-e^{-i \lambda \tau}\right)^{n} \frac{(1+i \lambda)^{n}}{(i \lambda)^{n}}: t<0\right\} \quad \text { and } \quad\left\{e^{i \lambda t}\left(1-e^{-i \lambda \tau}\right)^{n} \frac{(1+i \lambda)^{n}}{(i \lambda)^{n}}: t>T\right\} .
$$

It follows from formula (6) that we have one to one correspondence between elements $\xi^{(n)}(t, \tau)$ from the space $H$ and elements $e^{i \lambda t}\left(1-e^{-i \lambda \tau}\right)^{n} \frac{(1+i \lambda)^{n}}{(i \lambda)^{n}}$ from the space $L_{2}(f)$.

In terms of the defined Hilbert spaces the optimal linear estimate $\widehat{B}_{T} \xi$ is a projection of the element $B_{T} \xi$ of the space $H=L_{2}(\Omega, F, P)$ on the subspace $H^{0-}\left(\xi_{\tau}^{(n)}\right) \oplus H^{T+}\left(\xi_{-\tau}^{(n)}\right)=H^{0-}\left(\xi_{\tau}^{(n)}\right) \oplus H^{(T+\tau n)+}\left(\xi_{\tau}^{(n)}\right)$ generated by observations of the process $\xi(t)$. Due to the isometry between the spaces $H$ and $L_{2}(f)$ it is enough to find the spectral characteristic $h_{\tau}(\lambda) \in L_{2}^{0-}(f) \oplus L_{2}^{(T+\tau n)+}(f)$ of the projection $\widehat{B}_{T} \xi$. Properties of orthogonal projections in Hilbert spaces give us two conditions determining the spectral characteristic $h_{\tau}(\lambda)$ :

1) $h_{\tau}(\lambda) \in L_{2}^{0-}(f) \oplus L_{2}^{(T+\tau n)+}(f)$;

2) $\left(B_{T}^{\tau}(\lambda)\left(1-e^{-i \lambda \tau}\right)^{n} \frac{(1+i \lambda)^{n}}{(i \lambda)^{n}}-h_{\tau}(\lambda)\right) \perp L_{2}^{0-}(f) \oplus L_{2}^{(T+\tau n)+}(f)$, where

$$
B_{T}^{\tau}(\lambda)=\int_{0}^{T} b_{\tau}(t) e^{i \lambda t} d t
$$

The second condition implies that for all $t<0$ and for all $t>T+\tau n$ the function $h_{\tau}(\lambda)$ satisfies the equality

$$
\int_{-\infty}^{+\infty}\left(B_{T}^{\tau}(\lambda)\left(1-e^{-i \lambda \tau}\right)^{n} \frac{(1+i \lambda)^{n}}{(i \lambda)^{n}}-h_{\tau}(\lambda)\right) e^{-i \lambda t}\left(1-e^{i \lambda \tau}\right)^{n} \frac{(1-i \lambda)^{n}}{(-i \lambda)^{n}} f(\lambda) d \lambda=0 .
$$

Define the function

$$
C_{T}^{\tau}(\lambda)=\left(B_{T}^{\tau}(\lambda)\left(1-e^{-i \lambda \tau}\right)^{n} \frac{(1+i \lambda)^{n}}{(i \lambda)^{n}}-h_{\tau}(\lambda)\right) \frac{\left(1-e^{i \lambda \tau}\right)^{n}(1-i \lambda)^{n}}{(-i \lambda)^{n}} f(\lambda), \quad \lambda \in \mathbb{R}
$$

and its Fourier transformation

$$
\mathbf{c}_{\tau}(t)=\int_{-\infty}^{\infty} C_{T}^{\tau}(\lambda) e^{-i \lambda t} d \lambda, \quad t \in \mathbb{R} .
$$

It comes from condition (13) that the function $\mathbf{c}_{\tau}(t)$ equals to 0 outside the interval $[0 ; T+\tau n]$. Therefore,

$$
C_{T}^{\tau}(\lambda)=\int_{0}^{T+\tau n} \mathbf{c}_{\tau}(t) e^{i \lambda t} d t
$$

and the spectral characteristic $h_{\tau}(\lambda)$ of the estimate $\widehat{B}_{T} \xi$ can be represented as

$$
h_{\tau}(\lambda)=B_{T}^{\tau}(\lambda)\left(1-e^{-i \lambda \tau}\right)^{n} \frac{(1+i \lambda)^{n}}{(i \lambda)^{n}}-\frac{(-i \lambda)^{n} C_{T}^{\tau}(\lambda)}{(1-i \lambda)^{n}\left(1-e^{i \lambda \tau}\right)^{n} f(\lambda)} .
$$

Condition 1) implies the following representation of the spectral characteristic $h_{\tau}(\lambda)$ :

$$
h_{\tau}(\lambda)=h(\lambda)\left(1-e^{-i \lambda \tau}\right)^{n} \frac{(1+i \lambda)^{n}}{(i \lambda)^{n}}, \quad h(\lambda)=\int_{-\infty}^{0} s_{1}(t) e^{i \lambda t} d t+\int_{T+\tau n}^{\infty} s_{2}(t) e^{i \lambda t} d t,
$$

for some functions $s_{1}(t) \in L_{2}^{0-}$ and $s_{2}(t) \in L_{2}^{(T+\tau n)+}$ as well as conditions

$$
\int_{-\infty}^{+\infty}\left|h_{\tau}(\lambda)\right|^{2} f(\lambda) d \lambda<\infty, \quad \frac{(i \lambda)^{n} h_{\tau}(\lambda)}{(1+i \lambda)^{n}\left(1-e^{-i \lambda \tau}\right)^{n}} \in L_{2}^{0-} \oplus L_{2}^{(T+\tau n)+} .
$$


From this reasoning it follows that

$$
\int_{-\infty}^{+\infty}\left(B_{T}^{\tau}(\lambda)-\frac{\lambda^{2 n} C_{T}^{\tau}(\lambda)}{\left|1-e^{i \lambda \tau}\right|^{2 n}\left(1+\lambda^{2}\right)^{n} f(\lambda)}\right) e^{-i \lambda s} d \lambda=0, \quad s \in[0 ; T+\tau n] .
$$

Formula (10) determines a function $\mathbf{b}_{\tau}(t)=b_{\tau}(t)$ for $t \in[0 ; T]$. Put $\mathbf{b}_{\tau}(t)=0$ for $t \in(T ; T+\tau n]$. Then formula (14) implies the following equation that determines the unknown function $\mathbf{c}_{\tau}(t), t \in[0 ; T+\tau n]$ :

$$
\mathbf{b}_{\tau}(t)=\int_{0}^{T+\tau n} \mathbf{c}_{\tau}(s) \int_{-\infty}^{+\infty} e^{i \lambda(s-t)} \frac{\lambda^{2 n}}{\left|1-e^{i \lambda \tau}\right|^{2 n}\left(1+\lambda^{2}\right)^{n} f(\lambda)} d \lambda d s, t \in[0 ; T+\tau n] .
$$

Assume that the linear bounded operator $\mathbf{F}_{T+\tau n}^{\tau} \in L_{2}([0 ; T+\tau n])$ defined by the formula

$$
\left(\mathbf{F}_{T+\tau n}^{\tau} \mathbf{c}_{\tau}\right)(t)=\int_{0}^{T+\tau n} \mathbf{c}_{\tau}(s) \int_{-\infty}^{+\infty} \frac{e^{i \lambda(s-t)} \lambda^{2 n}}{\left|1-e^{i \lambda \tau}\right|^{2 n}\left(1+\lambda^{2}\right)^{n} f(\lambda)} d \lambda d s, \quad t \in[0 ; T+\tau n],
$$

has an inverse operator. In this case equation (15) has a solution

$$
\mathbf{c}_{\tau}(t)=\left(\left(\mathbf{F}_{T+\tau n}^{\tau}\right)^{-1} \mathbf{b}_{\tau}\right)(t), \quad t \in[0 ; T+\tau n],
$$

and the spectral characteristic $h_{\tau}(\lambda)$ of the optimal estimate $\widehat{B}_{T} \xi$ of the functional $B_{T} \xi$ is calculated by the formula

$$
\begin{gathered}
h_{\tau}(\lambda)=B_{T}^{\tau}(\lambda)\left(1-e^{-i \lambda \tau}\right)^{n} \frac{(1+i \lambda)^{n}}{(i \lambda)^{n}}-\frac{(-i \lambda)^{n} C_{T}^{\tau}(\lambda)}{\left(1-e^{i \lambda \tau}\right)^{n}(1-i \lambda)^{n} f(\lambda)}, \\
C_{T}^{\tau}(\lambda)=\int_{0}^{T+\tau n}\left(\left(\mathbf{F}_{T+\tau n}^{\tau}\right)^{-1} \mathbf{b}_{\tau}\right)(t) e^{i \lambda t} d t .
\end{gathered}
$$

The value of the mean-square error of the estimate is calculated by the formula

$$
\Delta\left(f, \widehat{B}_{T} \xi\right)=\frac{1}{2 \pi} \int_{-\infty}^{+\infty} \frac{\lambda^{2 n}\left|C_{T}^{\tau}(\lambda)\right|^{2}}{\left|1-e^{i \lambda \tau}\right|^{2 n}\left(1+\lambda^{2}\right)^{n} f(\lambda)} d \lambda=\left\langle\left(\mathbf{F}_{T+\tau n}^{\tau}\right)^{-1} \mathbf{b}_{\tau}, \mathbf{b}_{\tau}\right\rangle
$$

Summarizing our reasoning we come to conclusion that the following theorem holds true.

Theorem 2

Let $\{\xi(t), t \in \mathbb{R}\}$ be a continuous time random process with stationary $n$th increments $\xi^{(n)}(t, \tau)$ which has spectral density $f(\lambda)$ satisfying the minimality conditions (8). The optimal linear estimate $\widehat{B}_{T} \xi$ of the functional $B_{T} \xi$ which is determined by unknown values $\xi^{(n)}(t, \tau), t \in[0 ; T], \tau>0$, from observations of the process $\xi(t)$ at time points $t \in \mathbb{R} \backslash[0 ; T]$ is determined by formula (12). The spectral characteristic $h_{\tau}(\lambda)$ and the value of the mean-square error $\Delta\left(f, \widehat{B}_{T} \xi\right)$ of the optimal estimate $\widehat{B}_{T} \xi$ are calculated by formulas (16) and (17).

Formula (11) and Theorem 2 allow us to find the optimal estimate

$$
\widehat{A}_{T} \xi=-\int_{-\tau n}^{0} v_{\tau, T}(t) \xi(t) d t+\int_{-\infty}^{+\infty} h_{\tau}^{(a)}(\lambda) d Z(\lambda)
$$

of the functional $A_{T} \xi$. Define the function $\mathbf{a}(t)$ on the interval $[(0 ; T+\tau n]$ in the following way: $\mathbf{a}(t)=a(t)$, $t \in[0 ; T]$, and $\mathbf{a}(t)=0, t \in(T ; T+\tau n]$. After putting the function $\mathbf{b}_{\tau}(t)=\mathbf{D}_{T}^{\tau} \mathbf{a}(t), t \in[0 ; T+\tau n]$, into relations (16) and (17) we obtaine the following formulas for calculating the spectral characteristic and the value of the mean-square error of the estimate $\widehat{A}_{T} \xi$ :

$$
h_{\tau}^{(a)}(\lambda)=A_{T}(\lambda)\left(1-e^{-i \lambda \tau}\right)^{n} \frac{(1+i \lambda)^{n}}{(i \lambda)^{n}}-\frac{(-i \lambda)^{n} C_{T}^{\tau, a}(\lambda)}{\left(1-e^{i \lambda \tau}\right)^{n}(1-i \lambda)^{n} f(\lambda)},
$$


where

$$
\begin{gathered}
A_{T}(\lambda)=\int_{0}^{T} \mathbf{D}_{T}^{\tau} \mathbf{a}(t) e^{i \lambda t} d t, \quad C_{T}^{\tau, a}(\lambda)=\int_{0}^{T+\tau n}\left(\left(\mathbf{F}_{T+\tau n}^{\tau}\right)^{-1} \mathbf{D}_{T}^{\tau} \mathbf{a}\right)(t) e^{i \lambda t} d t \\
\Delta\left(f, \widehat{A}_{T} \xi\right)=\frac{1}{2 \pi} \int_{-\infty}^{+\infty} \frac{\lambda^{2 n}\left|C_{T}^{\tau, a}(\lambda)\right|^{2}}{\left|1-e^{i \lambda \tau}\right|^{2 n}\left(1+\lambda^{2}\right)^{n} f(\lambda)} d \lambda=\left\langle\left(\mathbf{F}_{T+\tau n}^{\tau}\right)^{-1} \mathbf{D}_{T}^{\tau} \mathbf{a}, \mathbf{D}_{T}^{\tau} \mathbf{a}\right\rangle .
\end{gathered}
$$

These reasons give us a possibility to conclude that the following theorem holds true.

\section{Theorem 3}

Let $\{\xi(t), t \in \mathbb{R}\}$ be a random process with stationary $n$th increments $\xi^{(n)}(t, \tau)$ which has spectral density $f(\lambda)$ satisfying the minimality conditions (8). Then the optimal linear estimate $\widehat{A}_{T} \xi$ of the functional $A_{T} \xi$ which depends on unknown values $\xi(t), t \in[0 ; T]$, from observations of the process $\xi(t)$ at time points $t \in \mathbb{R} \backslash[0 ; T]$ is determined by formula (18), where the function $v_{\tau, T}(t), t \in[-\tau n ; 0), \tau>0$, is calculated by formula (9). The spectral characteristic $h_{\tau}^{(a)}(\lambda)$ of the optimal estimate $\widehat{A}_{T} \xi$ is calculated by formula (19). The value of the meansquare error $\Delta\left(f, \widehat{A}_{T} \xi\right)$ of the estimate is calculated by formula (20).

As a matter of fact, Theorems 2 and 4 let us obtain more general result than the one given in Theorem 4 . Consider the functional

$$
A_{T+\tau n} \xi=\int_{0}^{T+\tau n} \mathbf{a}^{\alpha}(t) \xi(t) d t
$$

where the function $\mathbf{a}^{\alpha}(t)$ on the interval $[0 ; T+\tau n]$ is defined in the following way: $\mathbf{a}^{\alpha}(t)=a(t) t \in[0 ; T], a(t)$ is a given function, and $\mathbf{a}^{\alpha}(t)=\alpha(t), t \in(T ; T+\tau n], \alpha(t)$ is an arbitrary function. The following representations hold true:

$$
\begin{gathered}
A_{T+\tau n} \xi=B_{\tau, T+\tau n} \xi-V_{\tau, T+\tau n}= \\
=\int_{0}^{T+\tau n} b_{\tau, T+\tau n}(t ; \alpha) \xi^{(n)}(t, \tau) d t-\int_{-\tau n}^{0} v_{\tau, T+\tau n}(t ; \alpha) \xi(t) d t, \\
A_{T} \xi=-\int_{T}^{T+\tau n} \alpha(t) \xi(t) d t-\int_{-\tau n}^{0} v_{\tau, T+\tau n}(t ; \alpha) \xi(t) d t+B_{\tau, T+\tau n} \xi,
\end{gathered}
$$

where the functions $v_{\tau, T+\tau n}(t ; \alpha), t \in[0 ; T+\tau n]$, and $b_{\tau, T+\tau n}(t ; \alpha), t \in[0 ; T+\tau n]$, are defined by formulas (9) and (10). Since we know values of the process $\xi(t)$ at points $t \in[-\tau n ; 0)$ and $t \in(T ; T+\tau n]$, we have the following relation

$$
\Delta\left(f, \widehat{A}_{T} \xi\right)=\mathrm{E}\left|A_{T} \xi-\widehat{A}_{T} \xi\right|^{2}=\mathrm{E}\left|B_{T+\tau n} \xi-\widehat{B}_{T+\tau n} \xi\right|^{2}=\Delta\left(f, \widehat{B}_{T+\tau n} \xi\right) .
$$

For these reasons the optimal estimate $\widehat{A}_{T} \xi$ of the functional $A_{T} \xi$ can be calculated by the formula

$$
\widehat{A}_{T} \xi=-\int_{T}^{T+\tau n} \alpha(t) \xi(t) d t-\int_{-\tau n}^{0} v_{\tau, T+\tau n}(t ; \alpha) \xi(t) d t+\int_{-\infty}^{+\infty} h_{\tau}^{(a, \alpha)}(\lambda) d Z(\lambda) .
$$

The spectral characteristic $h_{\tau}^{(a, \alpha)}(\lambda)$ and the value of the mean-square error $\Delta\left(f, \widehat{A}_{T} \xi\right)$ of the optimal estimate $\widehat{A}_{T} \xi$ are calculated by the formulas

$$
h_{\tau}^{(a, \alpha)}(\lambda)=A_{T+\tau n}(\lambda)\left(1-e^{-i \lambda \tau}\right)^{n} \frac{(1+i \lambda)^{n}}{(i \lambda)^{n}}-\frac{(-i \lambda)^{n} C_{T+\tau n}^{\tau, a, \alpha}(\lambda)}{\left(1-e^{i \lambda \tau}\right)^{n}(1-i \lambda)^{n} f(\lambda)},
$$

where

$$
\begin{gathered}
A_{T+\tau n}(\lambda)=\int_{0}^{T+\tau n} \mathbf{D}_{T+\tau n}^{\tau} \mathbf{a}^{\alpha}(t) e^{i \lambda t} d t \\
C_{T+\tau n}^{\tau, a, \alpha}(\lambda)=\int_{0}^{T+\tau n}\left(\left(\mathbf{F}_{T+\tau n}^{\tau}\right)^{-1} \mathbf{D}_{T+\tau n}^{\tau} \mathbf{a}^{\alpha}\right)(t) e^{i \lambda t} d t,
\end{gathered}
$$




$$
\Delta\left(f, \widehat{A}_{T} \xi\right)=\frac{1}{2 \pi} \int_{-\infty}^{+\infty} \frac{\lambda^{2 n}\left|C_{T+\tau n}^{\tau, a, \alpha}(\lambda)\right|^{2}}{\left|1-e^{i \lambda \tau}\right|^{2 n}\left(1+\lambda^{2}\right)^{n} f(\lambda)} d \lambda=\left\langle\left(\mathbf{F}_{T+\tau n}^{\tau}\right)^{-1} \mathbf{D}_{T+\tau n}^{\tau} \mathbf{a}^{\alpha}, \mathbf{D}_{T+\tau n}^{\tau} \mathbf{a}^{\alpha}\right\rangle
$$

The following statement holds true.

\section{Theorem 4}

Let $\{\xi(t), t \in \mathbb{R}\}$ be a random process with stationary $n$th increments $\xi^{(n)}(t, \tau)$ which has spectral density $f(\lambda)$ satisfying the minimality conditions (8). For a given function $a(t), t \in[0 ; T]$, and arbitrary function $\alpha(t)$, $t \in(T ; T+\tau n], \tau>0$, the optimal linear estimate $\widehat{A}_{T} \xi$ of the functional $A_{T} \xi$ which depends on unknown values $\xi(t), t \in[0 ; T]$, from observations of the process $\xi(t)$ at time points $t \in \mathbb{R} \backslash[0 ; T]$ is determined by formula (21), where the function $v_{\tau, T+\tau n}(t), t \in[-\tau n ; 0)$, is calculated by formulas (9), (10). The spectral characteristic $h_{\tau}^{(a, \alpha)}(\lambda)$ of the optimal estimate $\widehat{A}_{T} \xi$ is calculated by formula (22). The value of the mean-square error $\Delta\left(f, \widehat{A}_{T} \xi\right)$ is calculated by formula (23).

\section{Minimax-robust method of interpolation}

The value of the mean-square error $\Delta\left(h_{\tau}^{(a, \alpha)}(f) ; f\right):=\Delta\left(f, \widehat{A}_{T} \xi\right)$ and the spectral characteristic $h_{\tau}^{(a, \alpha)}$ of the optimal estimate $\widehat{A}_{T} \xi$ of the functional $A_{T} \xi$ that depends on unknown values of the random process $\xi(t)$ with $n$th stationary increments can be calculated by formulas (22) and (23) if we know the spectral density $f(\lambda)$ of the random process $\xi(t)$. In practice, however, complete information on the spectral density of the random process is impossible in most cases, but, instead, we can obtain a set $\mathcal{D}$ of admissible spectral densities. In this case we can apply the minimax-robust method of estimation to the interpolation problem. This method gives us a possibility to find an estimate that minimizes the maximum of mean-square errors for all spectral densities from the given set $\mathcal{D}$ of admissible spectral densities simultaneously.

Definition 3

For a given class of spectral densities $\mathcal{D}$ the spectral densitiy $f_{0}(\lambda) \in \mathcal{D}$ is called least favourable in the class $\mathcal{D}$ for the optimal linear interpolation of the functional $A_{T} \xi$ if the following relation holds true

$$
\Delta\left(f_{0}\right)=\Delta\left(h_{\tau}^{(a, \alpha)}\left(f_{0}\right) ; f_{0}\right)=\max _{f \in \mathcal{D}} \Delta\left(h_{\tau}^{(a, \alpha)}(f) ; f\right) .
$$

\section{Definition 4}

For a given class of spectral densities $\mathcal{D}$ the spectral characteristic $h^{0}(\lambda)$ of the optimal linear estimate of the functional $A_{T} \xi$ is called minimax-robust if

$$
\begin{gathered}
h^{0}(\lambda) \in H_{\mathcal{D}}=\bigcap_{f \in \mathcal{D}} L_{2}^{0-}(f) \oplus L_{2}^{(T+\tau n)+}(f), \\
\min _{h \in H_{\mathcal{D}}} \max _{f \in \mathcal{D}} \Delta(h ; f)=\sup _{f \in \mathcal{D}} \Delta\left(h^{0} ; f\right) .
\end{gathered}
$$

The following lemma is a consequence of Theorem 4 and the former definition.

\section{Lemma 2}

Spectral density $f_{0} \in \mathcal{D}$ which satisfies the minimality conditions (8) is least favourable in the class $\mathcal{D}$ for the optimal linear interpolation of the functional $A_{T} \xi$ if the operator $\left(\mathbf{F}_{T+\tau n}^{\tau}\right)^{0}$ determined by the Fourier coefficients of the function

$$
\frac{\lambda^{2 n}}{\left|1-e^{i \lambda \tau}\right|^{2 n}\left(1+\lambda^{2}\right)^{n} f^{0}(\lambda)},
$$

is a solution of the conditional extremum problem

$$
\max _{f \in \mathcal{D}}\left\langle\left(\mathbf{F}_{T+\tau n}^{\tau}\right)^{-1} \mathbf{D}_{T+\tau n}^{\tau} \mathbf{a}^{\alpha}, \mathbf{D}_{T+\tau n}^{\tau} \mathbf{a}^{\alpha}\right\rangle=\left\langle\left(\left(\mathbf{F}_{T+\tau n}^{\tau}\right)^{0}\right)^{-1} \mathbf{D}_{T+\tau n}^{\tau} \mathbf{a}^{\alpha}, \mathbf{D}_{T+\tau n}^{\tau} \mathbf{a}^{\alpha}\right\rangle .
$$


Under the condition $h_{\tau}^{(a, \alpha)}\left(f_{0}\right) \in H_{\mathcal{D}}$ the minimax-robust spectral characteristic of the optimal linear estimate of the functional $A_{T} \xi$ is defined by the relation $h^{0}=h_{\tau}^{(a, \alpha)}\left(f_{0}\right)$.

The minimax-robust spectral characteristic $h^{0}$ and least favourable spectral density $f_{0}$ form a saddle point of the function $\Delta(h ; f)$ on the set $H_{\mathcal{D}} \times \mathcal{D}$. The saddle point inequalities

$$
\Delta\left(h ; f_{0}\right) \geq \Delta\left(h^{0} ; f_{0}\right) \geq \Delta\left(h^{0} ; f\right) \quad \forall f \in \mathcal{D}, \forall h \in H_{\mathcal{D}}
$$

hold true if $h^{0}=h_{\tau}^{(a, \alpha)}\left(f_{0}\right)$ and $h_{\tau}^{(a, \alpha)}\left(f_{0}\right) \in H_{\mathcal{D}}$, where the function $f_{0}$ is a solution of the conditional extremum problem

$$
\begin{gathered}
\widetilde{\Delta}(f)=-\Delta\left(h_{\tau}^{(a, \alpha)}\left(f_{0}\right) ; f\right) \rightarrow \inf , \quad f \in \mathcal{D}, \\
\Delta\left(h_{\tau}^{(a, \alpha)}\left(f_{0}\right) ; f\right)= \\
=\frac{1}{2 \pi} \int_{-\infty}^{+\infty}\left|\int_{0}^{T+\tau n}\left(\left(\left(\mathbf{F}_{T+\tau n}^{\tau}\right)^{0}\right)^{-1} \mathbf{D}_{T+\tau n}^{\tau} \mathbf{a}^{\alpha}\right)(t) e^{i \lambda t} d t\right|^{2} \frac{\lambda^{2 n} f_{0}^{-2}(\lambda)}{\left|1-e^{i \lambda \tau}\right|^{2 n}\left(1+\lambda^{2}\right)^{n}} f(\lambda) d \lambda .
\end{gathered}
$$

Conditional extremum problem (25) is equivalent to the unconditional extremum problem

$$
\Delta_{\mathcal{D}}(f)=\widetilde{\Delta}(f)+\delta(f \mid \mathcal{D}) \rightarrow \inf ,
$$

where $\delta(f \mid \mathcal{D})$ is the indicator function of the set $\mathcal{D}$. Solution $f_{0}$ to this unconditional extremum problem is characterized by the condition $0 \in \partial \Delta_{\mathcal{D}}\left(f_{0}\right)$ [32], [29], where $\partial \Delta_{\mathcal{D}}\left(f_{0}\right)$ is a subdifferential of the functional $\Delta_{\mathcal{D}}(f)$. In the following sections we obtained formulas which gives us a method of description of the least favourable spectral densities in some classes of admissible densities.

\section{Least favourable spectral density in the class $\mathcal{D}_{0}$}

Consider the following class of admissible spectral densities

$$
\mathcal{D}_{0}=\left\{f(\lambda) \mid \frac{1}{2 \pi} \int_{-\infty}^{+\infty} f(\lambda) d \lambda \leq P\right\}
$$

Let us assume that $f_{0}(\lambda) \in \mathcal{D}_{0}$ and the function

$$
h_{f}\left(f_{0}\right)=\left|\int_{0}^{T+\tau n}\left(\left(\left(\mathbf{F}_{T+\tau n}^{\tau}\right)^{0}\right)^{-1} \mathbf{D}_{T+\tau n}^{\tau} \mathbf{a}^{\alpha}\right)(t) e^{i \lambda t} d t\right| \frac{\lambda^{n} f_{0}^{-1}(\lambda)}{\left|1-e^{i \lambda \tau}\right|^{n}|1+i \lambda|^{n}}
$$

is bounded. Then the condition $0 \in \partial \Delta_{\mathcal{D}}\left(f_{0}\right)$ obtained in the previous section implies the following equality which determines the least favourable spectral density:

$$
\left|\int_{0}^{T+\tau n}\left(\left(\left(\mathbf{F}_{T+\tau n}^{\tau}\right)^{0}\right)^{-1} \mathbf{D}_{T+\tau n}^{\tau} \mathbf{a}^{\alpha}\right)(t) e^{i \lambda t} d t\right|=\beta f_{0}(\lambda)|\lambda|^{-n}\left|1-e^{i \lambda \tau}\right|^{n}|1+i \lambda|^{n},
$$

where $\beta \geq 0$ is a constant such that $\beta \neq 0$ in the case where $\int_{-\infty}^{+\infty} f_{0}(\lambda) d \lambda=2 \pi P$.

Summing up the results described above we can formulate the following statement.

Theorem 5

Let spectral density $f_{0}(\lambda) \in \mathcal{D}_{0}$ satisfy the minimality conditions (8) and let the function $h_{f}\left(f_{0}\right)$ defined by formula (26) be bounded. Then for a given function $a(t), t \in[0 ; T]$ and an arbitrary function $\alpha(t), t \in(T ; T+\tau n]$, the spectral density $f_{0}(\lambda)$ is least favourable in the class $\mathcal{D}_{0}$ for the optimal interpolation of the functional $A_{T} \xi$ from 
observations of the random process $\xi(t)$ at time points $t \in \mathbb{R} \backslash[0 ; T]$ if it is of the form

$$
f_{0}(\lambda)=\frac{|\lambda|^{n} \beta^{-1}\left|C_{T+\tau n}^{\tau, a, \alpha}(\lambda)\right|}{\left|1-e^{i \lambda \tau}\right|^{n}|1+i \lambda|^{n}}
$$

and $f_{0}(\lambda)$ is a solution to conditional extremum problem (24). The minimax-robust spectral characteristic $h^{0}=$ $h_{\tau}^{(a, \alpha)}\left(f_{0}\right)$ of the optimal estimate $\widehat{A}_{T} \xi$ of the functional $A_{T} \xi$ is calculated by formula (22).

\section{Least favourable spectral density in the class $\mathcal{D}_{v}^{u}$}

In this section let us consider the minimax-robust interpolation problem for the functional $A_{T} \xi$ in the case where the class of admissible spectral densities is defined as follows:

$$
\mathcal{D}_{v}^{u}=\left\{f(\lambda) \mid v(\lambda) \leq f(\lambda) \leq u(\lambda), \frac{1}{2 \pi} \int_{-\infty}^{+\infty} f(\lambda) d \lambda \leq P\right\}
$$

where spectral densities $u(\lambda)$ and $v(\lambda)$ are known, fixed and bounded.

Let us assume that the spectral density $f_{0}(\lambda) \in \mathcal{D}_{v}^{u}$ determines a bounded function $h_{f}\left(f_{0}\right)$ by formula (26). It comes from the condition $f_{0} \in \partial \Delta_{\mathcal{D}}\left(f_{0}\right)$ that the least favourable spectral density satisfies the equation

$$
\left|\int_{0}^{T+\tau n}\left(\left(\left(\mathbf{F}_{T+\tau n}^{\tau}\right)^{0}\right)^{-1} \mathbf{D}_{T+\tau n}^{\tau} \mathbf{a}^{\alpha}\right)(t) e^{i \lambda t} d t\right|=\frac{f_{0}(\lambda)\left(\gamma_{1}(\lambda)+\gamma_{2}(\lambda)+\beta\right)}{|\lambda|^{n}\left|1-e^{i \lambda \tau}\right|^{-n}|1+i \lambda|^{-n}},
$$

where $\gamma_{1}(\lambda) \leq 0$ and $\gamma_{1}(\lambda)=0$ in the case where $f_{0}(\lambda) \geq v(\lambda) ; \gamma_{2}(\lambda) \geq 0$ and $\gamma_{2}(\lambda)=0$ in the case where $f_{0}(\lambda) \leq u(\lambda)$.

The following statement holds true.

\section{Theorem 6}

Let spectral density $f_{0}(\lambda) \in \mathcal{D}_{v}^{u}$ satisfy the minimality conditions (8) and let the function $h_{f}\left(f_{0}\right)$ determined by formula (26) be bounded. Then for a given function $a(t), t \in[0 ; T]$ and an arbitrary function $\alpha(t), t \in(T ; T+\tau n]$, the spectral density $f_{0}(\lambda)$ is least favourable in the class $\mathcal{D}_{v}^{u}$ for the optimal interpolation of the functional $A_{T} \xi$ from observations of the random process $\xi(t)$ at time points $t \in \mathbb{R} \backslash[0 ; T]$ if it is of the form

$$
f_{0}(\lambda)=\max \left\{v(\lambda), \min \left\{u(\lambda), \frac{|\lambda|^{n} \beta^{-1}\left|C_{T+\tau n}^{\tau, a, \alpha}(\lambda)\right|}{\left|1-e^{i \lambda \tau}\right|^{n}|1+i \lambda|^{n}}\right\}\right\}
$$

and $f_{0}(\lambda)$ is a solution to conditional extremum problem (24). The minimax-robust spectral characteristic $h^{0}=$ $h_{\tau}^{(a, \alpha)}\left(f_{0}\right)$ of the optimal estimate $\widehat{A}_{T} \xi$ of the functional $A_{T} \xi$ is calculated by formula (22).

\section{Least favourable spectral density in the class $\mathcal{D}_{2 \varepsilon}$}

Consider another example of a class of admissible spectral densities, that is the class of densities that describe an " $\varepsilon-$ neighbourhood" in the space $L_{2}$ of a given spectral density $f_{1}(\lambda)$ :

$$
\mathcal{D}_{2 \varepsilon}=\left\{f(\lambda)\left|\frac{1}{2 \pi} \int_{-\infty}^{+\infty}\right| f(\lambda)-\left.f_{1}(\lambda)\right|^{2} d \lambda \leq \varepsilon\right\} .
$$

Let a spectral density $f_{0}(\lambda) \in \mathcal{D}_{2 \varepsilon}$ be such that the function determined by formula (26) is bounded. The condition $f_{0} \in \partial \Delta_{\mathcal{D}}\left(f_{0}\right)$ gives us the equation

$$
\left|\int_{0}^{T+\tau n}\left(\left(\left(\mathbf{F}_{T+\tau n}^{\tau}\right)^{0}\right)^{-1} \mathbf{D}_{T+\tau n}^{\tau} \mathbf{a}^{\alpha}\right)(t) e^{i \lambda t} d t\right|^{2}=\frac{\beta f_{0}^{2}(\lambda)\left(f_{0}(\lambda)-f_{1}(\lambda)\right)}{|\lambda|^{n}\left|1-e^{i \lambda \tau}\right|^{-n}|1+i \lambda|^{-n}},
$$


where $\beta$ is a constant. The derived equation (28), extremum condition (24) and the condition

$$
\frac{1}{2 \pi} \int_{-\infty}^{+\infty}\left|f_{0}(\lambda)-f_{1}(\lambda)\right|^{2} d \lambda=\varepsilon
$$

determine the least favourable spectral density $f_{0}(\lambda) \in \mathcal{D}_{2 \varepsilon}$. Let us present this result as the following statement.

\section{Theorem 7}

Let spectral density $f_{0}(\lambda) \in \mathcal{D}_{2 \varepsilon}$ satisfy the minimality conditions $(8)$ and let the function $h_{f}\left(f_{0}\right)$ determined by formula (26) be bounded. Then for a given function $a(t), t \in[0 ; T]$ and an arbitrary function $\alpha(t), t \in(T ; T+\tau n]$, the spectral density $f_{0}(\lambda)$ is least favourable in the class $\mathcal{D}_{2 \varepsilon}$ for the optimal interpolation of the functional $A_{T} \xi$ from observations of the random process $\xi(t)$ at time points $t \in \mathbb{R} \backslash[0 ; T]$ if it satisfies relations (28), (29) and determines a solution to conditional extremum problem (24). The minimax-robust spectral characteristic $h^{0}=h_{\tau}^{(a, \alpha)}\left(f_{0}\right)$ of the optimal estimate $\widehat{A}_{T} \xi$ of the functional $A_{T} \xi$ is calculated by formula $(22)$.

\section{Conclusions}

In this article we proposed a solution of the problem of mean-square optimal estimation of the linear functional $A_{T} \xi=\int_{0}^{T} a(t) \xi(t) d t$ which depends on the unknown (missed) values of a random process $\xi(t)$ with $n$th stationary increments from observations of this process $\xi(t)$ at time points $t \in \mathbb{R} \backslash[0 ; T]$. Using Kolmogorov's Hilbert space projection method we derived formulas for calculating the value of the mean-square error and the spectral characteristic of the optimal estimate of the functional $A_{T} \xi$ under the condition that spectral density of the process is exactly known. In the case where spectral density is not exactly known, but a set of admissible spectral densities is available, we applied the minimax-robust method of interpolation which provided us relations determining least favourable spectral densities and minimax-robust spectral characteristics of the optimal estimates of the functional $A_{T} \xi$ for different classes of spectral densities.

\section{REFERENCES}

1. I. I. Gikhman and A. V. Skorokhod, The theory of stochastic processes. I., Berlin: Springer, 2004.

2. I. I. Dubovets'ka, O.Yu. Masyutka and M.P. Moklyachuk, Interpolation of periodically correlated stochastic sequences, Theory Probab. Math. Stat., vol. 84, pp. 43-56, 2012.

3. I. I. Dubovets'ka and M. P. Moklyachuk, Filtration of linear functionals of periodically correlated sequences, Theory Probab. Math. Stat., vol. 86, pp. 51-64, 2013.

4. I. I. Dubovets'ka and M. P. Moklyachuk, Extrapolation of periodically correlated processes from observations with noise, Theory Probab. Math. Stat., vol. 88, pp. 67-83, 2014.

5. I. I. Dubovets'ka and M. P. Moklyachuk, Minimax estimation problem for periodically correlated stochastic processes, Journal of Mathematics and System Science, vol. 3, no. 1, pp. 26-30, 2013.

6. I. I. Dubovets'ka and M. P. Moklyachuk, On minimax estimation problems for periodically correlated stochastic processes, Contemporary Mathematics and Statistics, vol.2, no. 1, pp. 123-150, 2014.

7. U. Grenander, A prediction problem in game theory, Arkiv för Matematik, vol. 3, pp. 371-379, 1957.

8. J. Franke, Minimax robust prediction of discrete time series, Z. Wahrscheinlichkeitstheor. Verw. Gebiete, vol. 68, pp. 337-364, 1985.

9. J. Franke and H. V. Poor, Minimax-robust filtering and finite-length robust predictors, Robust and Nonlinear Time Series Analysis. Lecture Notes in Statistics, Springer-Verlag, vol. 26, pp. 87-126, 1984.

10. K. Karhunen, Über lineare Methoden in der Wahrscheinlichkeitsrechnung, Annales Academiae Scientiarum Fennicae. Ser. A I, vol. 37, 1947.

11. S. A. Kassam and H. V. Poor, Robust techniques for signal processing: A survey, Proceedings of the IEEE, vol. 73, no. 3, pp. 433-481, 1985.

12. A. N. Kolmogorov, Selected works by A. N. Kolmogorov. Vol. II: Probability theory and mathematical statistics. Ed. by A. N. Shiryayev. Mathematics and Its Applications. Soviet Series. 26. Dordrecht etc. Kluwer Academic Publishers, 1992.

13. M. M. Luz and M. P. Moklyachuk, Interpolation of functionals of stochastic sequences with stationary increments, Theory Probab. Math. Stat., vol. 87, pp. 117-133, 2013.

14. M. M. Luz and M. P. Moklyachuk, Interpolation of functionals of stochastic sequences with stationary increments for observations with noise, Prykl. Stat., Aktuarna Finans. Mat., no. 2, pp. 131-148, 2012.

15. M. M. Luz and M. P. Moklyachuk, Minimax-robust filtering problem for stochastic sequence with stationary increments, Theory Probab. Math. Stat., vol. 89, pp. 127-142, 2014. 
16. M. Luz and M. Moklyachuk, Robust extrapolation problem for stochastic processes with stationary increments, Mathematics and Statistics, vol. 2, no. 2, pp. 78-88, 2014.

17. M. Luz and M. Moklyachuk, Minimax-robust filtering problem for stochastic sequences with stationary increments and cointegrated sequences, Statistics, Optimization \& Information Computing, vol. 2, no. 3, pp. 176-199, 2014.

18. M. Moklyachuk and M. Luz, Robust extrapolation problem for stochastic sequences with stationary increments, Contemporary Mathematics and Statistics, vol. 1, no. 3, pp. 123-150, 2013

19. M. P. Moklyachuk, Minimax extrapolation and autoregressive-moving average processes, Theory Probab. Math. Stat., vol. 41, pp. 77-84, 1990.

20. M. P. Moklyachuk, Robust procedures in time series analysis, Theory of Stochastic Processes, vol. 6, no. 3-4, pp. 127-147, 2000.

21. M. P. Moklyachuk, Game theory and convex optimization methods in robust estimation problems, Theory of Stochastic Processes, vol. 7, no. 1-2, pp. 253-264, 2001.

22. M. P. Moklyachuk, Robust estimations of functionals of stochastic processes, Kyiv University, Kyiv, 2008.

23. M. Moklyachuk and A. Masyutka, Extrapolation of multidimensional stationary processes, Random Operators and Stochastic Equations, vol. 14, no. 3, pp.233-244, 2006.

24. M. Moklyachuk and A. Masyutka, Robust estimation problems for stochastic processes, Theory of Stochastic Processes, vol. 12, no. 3-4, pp. 88-113, 2006.

25. M. Moklyachuk and A. Masyutka, Robust filtering of stochastic processes, Theory of Stochastic Processes, vol. 13, no. 1-2, pp. 166-181, 2007.

26. M. Moklyachuk and A. Masyutka, Minimax prediction problem for multidimensional stationary stochastic sequences, Theory of Stochastic Processes, vol. 14, no. 3-4, pp. 89-103, 2008.

27. M. Moklyachuk and A. Masyutka, Minimax prediction problem for multidimensional stationary stochastic processes, Communications in Statistics - Theory and Methods., vol. 40, no. 19-20, pp. 3700-3710, 2001.

28. M. Moklyachuk and O. Masyutka, Minimax-robust estimation technique for stationary stochastic processes, LAP LAMBERT Academic Publishing, 2012.

29. M. P. Moklyachuk, Nonsmooth analysis and optimization, Kyiv University, Kyiv, 2008

30. M. S. Pinsker and A. M. Yaglom, On linear extrapolation of random processes with nth stationary increments, Doklady Akademii Nauk SSSR, vol. 94, pp. 385-388, 1954

31. M. S. Pinsker, The theory of curves with nth stationary increments in Hilber spaces, Izvestiya Akademii Nauk SSSR. Ser. Mat., vol. 19, no. 5, pp. 319-344, 1955.

32. B. N. Pshenichnyi, Necessary conditions of an extremum, "Nauka", Moskva, 1982.

33. Yu. A. Rozanov, Stationary stochastic processes. 2nd rev. ed., "Nauka", Moskva, 1990.

34. K. S. Vastola and H. V. Poor, An analysis of the effects of spectral uncertainty on Wiener filtering, Automatica, vol. 28, pp. 289-293, 1983.

35. N. Wiener, Extrapolation, interpolation and smoothing of stationary time series. With engineering applications, The M. I. T. Press, Massachusetts Institute of Technology, Cambridge, Mass., 1966.

36. A. M. Yaglom, Correlation theory of stationary and related random functions. Vol. 1: Basic results, Springer Series in Statistics, Springer-Verlag, New York etc., 1987.

37. A. M. Yaglom, Correlation theory of stationary and related random functions. Vol. 2: Supplementary notes and references, Springer Series in Statistics, Springer-Verlag, New York etc., 1987.

38. A. M. Yaglom, Correlation theory of stationary and related random processes with stationary nth increments, Mat. Sbornik, vol 37, no. 1, pp. 141-196, 1955.

39. A. M. Yaglom, Some classes of random fields in n-dimensional space related with random stationary processes, Teor. Veroyatn Primen., vol. 11, no. 3, pp. 292-337, 1957. 